\title{
Silent brain infarctions and leuko-araiosis in Chinese patients with first-ever acute lacunar strokes
}

\author{
Peterus Thajeb ${ }^{1,2,3,4^{*}}$, Wen-Yuan Lee $^{2}$, Chung-Hung Shih ${ }^{5}$, Teguh Thajeb ${ }^{6}$, James Davis ${ }^{3}$, Rosanne \\ Harrigan $^{3}$, Linda Chang ${ }^{3}$
}

${ }^{1}$ Center for Stroke Care and Prevention, Cathay General Hospital Sijhih, Sijhih, Taiwan, China;

${ }^{2}$ Section of Neurosurgery and Neurology, China Medical University Hospital, Taipei, Taiwan, China;

${ }^{3}$ Biomedical Science, Graduate Division, John A Burns School of Medicine, University of Hawaii at Manoa, Hawaii, USA;

${ }^{4}$ Faculty of Medicine, National Yang-Ming University, Taipei, Taiwan, China;

${ }^{5}$ Institute of Respiratory Therapy, Taipei Medical University, Taipei, Taiwan, China;

${ }^{6}$ Department of Internal Medicine, Landseed Hospital, Pingjen, Taiwan, China.

Email: peterus@hawaii.edu; thajebp@hotmail.com

Received 3 January 2010; revised 12 January 2010; accepted 15 January 2010.

\begin{abstract}
We report on silent brain infarction (SBI) and leukoaraiosis (LA) of 23 patients with clinically diagnosed "first-ever" acute ischemic lacunar stroke. The lacunar syndromes were pure motor hemiparesis (10), pure sensory syndrome (2), ataxic hemiparesis (3), dysarthria clumsy hand syndrome (3), and sensorymotor deficit (5). Nineteen out of the 23 patients presented with completed strokes on arrival to the hospital, and $4(17 \%)$ developed evolving-stroke within 24 hours of stroke onset. A lacune corresponded to the acute stroke could be found in all patients on brain magnetic resonance imaging (MRI), and in 18 (78\%) on brain computed tomography (CT). MRI showed additional subclinical or asymptomatic "silent brain infarctions or lacunes" (SBI) in 19 (83\%) of 23 patients, and leuko-araiosis (LA) of moderate to severe degree ( $\geq$ grade 2 ) was present in $61 \%$ of patients although dementia was absent. Hypertension is the risk factor in $78 \%$ of cases followed by diabetes mellitus, smoking, and elevated plasma cholesterol level. Independence of the types of lacunar syndromes, patients with hypertension and diabetes mellitus are associated with high grade LA. None with normal blood pressure and plasma glucose had grade 3 or grade 4 LA (p < 0.05). In conclusion, evolving-stroke occurs in onefifth of patients with "first-ever" lacunar infarct within the first 24 hours of stroke onset. SBI was found in $83 \%$ of cases. Hypertension and diabetes mellitus are associated with additional SBI and high grade LA. The severity of leuko-araiosis per se dictates the cerebrovascular risks.
\end{abstract}

Keywords: Computed Tomography; First-Ever Stroke; Lacune; Leuko-Araiosis; Magnetic Resonance Imaging;
MRI; Silent Brain Infarction

\section{INTRODUCTION}

Elegant study on 1042 routine autopsied brains by Fisher in 1965 [1,2], and data from 2,859 necropsied cases reported by Tuszynski et al. [3] suggest that lacunes occur in between $6 \%$ [3] and $11 \%$ [1,2] of cases, respectively. Approximately, eighty percent of these lacunes had no history of stroke and neurological deficit, and thus called "asymptomatic" or "silent" lacunes $[3,4]$. During life, the diagnosis of asymptomatic or subclinical silent lacunes in the pre-era of MRI has been a difficult task for several reasons. First, ethically a neurological healthy subject would not justify having an intensive laboratory work-up for any possible reason of unexpected intracranial silent lesion. Second, in the earlier days, before the advent of MRI technique, a brain CT would not sensitive enough to detect a small lesion of less than $15 \mathrm{~mm}$ in a clinically overt lacunar stroke. Therefore, the actual incidence of subclinical silent lacunes in the general population, and in asymptomatic patients with cerebrovascular risk factors remains conjectural. Extensive use of brain MRI in the past decade had suggested that prevalence of MRI-based diagnosis of silent brain infarction (SBI) ranged from $8 \%$ [5] to $28 \%$ [6]. These SBIs included silent lacunes and silent non-lacunar infarctions [5-8]. A screening health examination in Japan on 246 neurologically normal adults revealed 13\% had possible silent lacunar lesions on brain MRI [9]. But nothing is known about SBI in Chinese/Taiwanese with "first-ever" acute ischemic lacunar syndromes. This issue will be addressed herein.

\section{PATIENTS AND METHODS}

Patients recruited in this study were a subset from the 
authors' consecutive stroke registry between January 1, 2007 and December 31, 2008. Subjects with a lacunar infarction due to small penetrating artery occlusion are often considered "minor" stroke by both laymen and many clinicians. Good recovery of symptoms and signs in certain proportion of cases leads to underestimation of the prevalence of lacunar infarction and silent brain infarction. In order to obtain a homogeneous study population, we address the question that how often a silent brain infarction (SBI) can be seen in patients with clinically "first-ever" lacunar stroke? Therefore, patients with non-lacunar infarction were excluded from the study. Only those fulfilling all diagnostic criteria set below for acute first-ever lacunar infarction and those with complete clinical data and available brain CT and MRI within 5 days of stroke onset will be analyzed. The diagnostic criteria of "first-ever" acute lacunar infarction were: 1) acute stroke with clinical manifestation of one of the 5 typical clinical lacunar syndromes described by Fisher $[1,2]$; 2) absence of past history of presumed stroke or transient ischemic attack (TIA), including amourosis fugax; 3) normal electroencephalography (EEG); 4) neuroimaging studies (brain MRI or CT or both) showing a corresponded acute brain infarction in the territory of the penetrating artery and of the size of a "lacune" (diameter of $15 \mathrm{~mm}$ or less); and 5) exclusion of those with presumed vascular dementia on mini-mental state examination (MMSE) [10] or modified mini-mental test (MMT) [11], or both, and on Hatchinski ischemic score [12]. Twenty-three patients thus recruited consisted of 6 women and 17 men. The age at stroke onset ranged from 51 to 85 years (mean 65 years).

MRI was performed by using either one of the following protocol. The old MRI machine (0.5 Tesla superconductive unit, MRI-50 A, manufactured by Toshiba Corporation) was a multiple-spin-echo T1-weighted image with TR $500 \mathrm{msec} / \mathrm{TE} 15 \mathrm{msec}$, T2-weighted images with TR $3000 \mathrm{msec} / \mathrm{TE} 120 \mathrm{msec}$, and proton density images (PDI) with TR $3000 \mathrm{msec} / \mathrm{TE} 30 \mathrm{msec}$. The lesions could be round or oval in shape. The single lesion that matched completely to the clinical presentation (one of the 5 lacunar syndromes) was called the corresponding lesion. Other unexpected additional (old) lesions or lacunes were called additional lesions or "silent" lacunes. A smaller "dot-like", or spotty high signal intensity on T2WI and PDI at the cerebal subcortical region that beyond the territory of the penetrating arteries, may or may not be seen on T1WI MRI or brain CT were considered "etat crible" [13]. The hyperintense poorly demarcated pathches of irregular border surrounding the lateral ventricles were designated as "leuko-araiosis" (LA) or periventricular "caps" or "rims" [14]. The grading of severity of LA was as follows: LA grade 0 (no leuko-araiosis), LA grade 1 (mild leuko-araiosis at the frontal horn subcortical white matter), LA grade 2 (moderate LA involv- ing the frontal and posterior horn white matter), LA grade 3 (severe LA involving the whole rims of lateral ventricular white matter), and LA grade 4 (advanced LA, grade 3 LA plus involvement of the centrum semiovale). The new MRI machine has T1WI [TI 860.0/TR 2,200.0/TE 11.0], T2WI [TR 3,300.0/TE 113.0], FLAIR [TI 2,500.0/TR 9,000.0/TE 113.0], diffusion weighted images (DWI) [TR 3,600.0/TE 84.0], ADC map, and magnetic resonance angiogram (MRA) [TR 37.0/TE 7.0]. MRI definition of a lacune remains the same for T1WI and T2WI. Additional imaging parameters for acute lacunar infarction were lesion of high signal on T2FLAIR, and DWI, and low signal on ADC map. Asymptomatic or subclinical "silent" (old) cerebral infarctions were characterized by signal intensity of iso-or low-signal on DWI and iso-or high signal on ADC map.

Statistical analyses: Categorical Chi-square or Fisher's exact test (for $\mathrm{n} \leq 5$ ) were used for statistical analyses. Probability of $<0.05$ was considered statistical significance.

\section{RESULTS}

Mean age at onset was 65 years (range 51 to 85 years) with men to women ratio of 2.8 . There was no statistical difference between gender and types of lacunar syndromes. Additional lesions suggesting silent cerebral lacunar infarctions were encountered in $83 \%$ of patients on MRI (Table 1) as compared to $43 \%$ on CT $(\mathrm{p}<0.005)$. Hypertension was top one risk factor in $78 \%$ of patients. Other risk factors were diabetes mellitus (11 cases,) (48\%), high cholesterol level (> $200 \mathrm{mg} / \mathrm{dL}$ ) or/and low HDL-cholesterol level (10 cases), smoking (10 cases), hyperfibrinogenemia (> $400 \mathrm{mg} / \mathrm{dL}$ ) (6 cases), high hematocrit (4), and hyperuricemia (> $7.5 \mathrm{mg} / \mathrm{dL}$ ) (4 cases). The initial manifestations of lacunar syndromes were pure motor hemiparesis (PMH) in 10 patients, sensoymotor deficit (SM) in 5, ataxic hemiparesis ( $\mathrm{AH})$ in 3 , dysarthria clumsy hand syndrome (DCH) in 3 , and pure sensory syndrome (PS) in 2. Nineteen of the 23 patients presented with completed strokes on arrival to the hospital. Four patients (17\%) developed evolving stroke in the subsequent 24 hours of stroke onset. The frequencies of abnormalities found on brain CT and MRI were shown in Table 1. Hyperintense lesions on T2WI and PDI of brain MRI included the lacunes and etat cribles, were most commonly encountered at the corona radiata or periventricular white matter (PVWM), followed by internal

Table 1. Frequencies of abnormalities found on brain CT and MRI of 23 patients with "first-ever" lacunar infarction.

\begin{tabular}{lcc}
\hline \multicolumn{3}{c}{ Number $(\%)$ of Patients } \\
\hline $\begin{array}{l}\text { Detectable abnormalities } \\
\text { Corresponding single lesion }\end{array}$ & Brain CT & Brain MRI \\
of acute infarction & $18(78)$ & $23(100)$ \\
Additional lesions & $10(43)$ & $19(83)^{*}$ \\
Leuko-araiosis & $2(9)$ & $14(61)^{*}$ \\
$* \mathrm{p}<0.05$ & &
\end{tabular}


capsule, basal ganglia, thalamus, and brain stem (Table 2 and Figure 1). Severity of leuko-araiosis in different subgroups was shown in Table 3. Regardless of the types of lacunar syndromes, patients with hypertension or diabetes mellitus were likely having moderate to severe LA (grade 2 to 3 ) (13 of the 18 patients) $(72 \%$, in contrast to $20 \%$ for those without these risk factors). Combination of hypertension and DM strengthened this effect from severe to advanced LA (grade 3 to 4). None of patient with normotension and euglycemia had LA > grade $2(\mathrm{p}<0.05)$.

Table 2. Frequency and locations of additional silent lacunes and etat crible in 23 patients with various types of acute firstever brain lacunar infarctions.

\begin{tabular}{|c|c|c|c|c|c|}
\hline \multicolumn{6}{|c|}{ Location of Silent Number (\%) of Patients with Positive CT/MRI } \\
\hline \multirow[t]{2}{*}{ Lacunes } & $\mathrm{PMH}$ & $\mathrm{SM}$ & $\mathrm{AH}$ & $\mathrm{DCH}$ & PS \\
\hline & $(10)$ & $(5)$ & (3) & (3) & (2) \\
\hline Corona radiata* & $4 / 7$ & $2 / 3$ & $0 / 1$ & $0 / 2$ & $1 / 2$ \\
\hline Internal capsule & $3 / 5$ & $2 / 2$ & $1 / 2$ & $2 / 3$ & $0 / 1$ \\
\hline Basal ganglia & $1 / 4$ & $0 / 2$ & $1 / 1$ & $0 / 0$ & $0 / 0$ \\
\hline Thalamus & $0 / 2$ & $2 / 3$ & $0 / 1$ & $1 / 1$ & $1 / 1$ \\
\hline Brainstem & $1 / 3$ & $0 / 2$ & $1 / 2$ & $0 / 1$ & $0 / 0$ \\
\hline
\end{tabular}

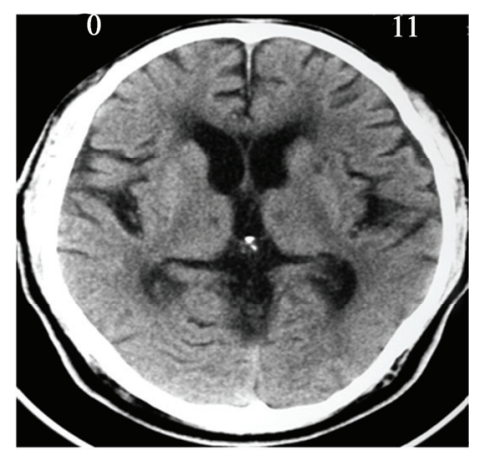

(a)

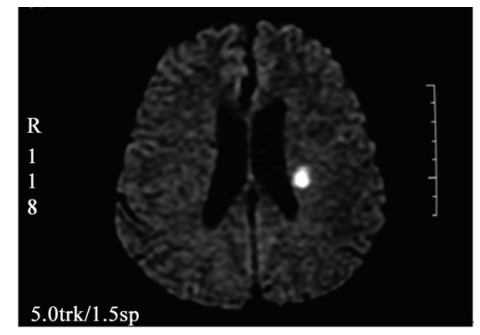

(b)

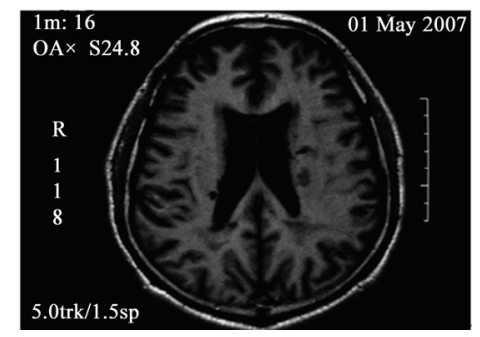

(c)

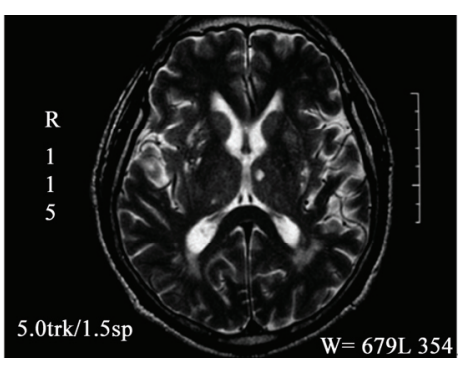

(d)

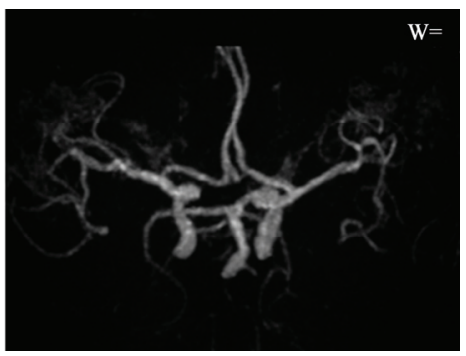

(e)

Figure 1. (a) Brain CT of an acute lacunar infarction involving the left thalamus, and old silent lacunes at left anterior basal ganglion, and right thalamus; (b-e) Brain MRI of another patient with acute lacunar infarction involving the left internal capsule; (b) Diffusion-weighted images (DWI) [TR 3,600.0/TE 84.0]; (c) T1WI; (d) T2WI show additional multiple old "silent brain infarctions" involving bilateral paraventricular white matter/corona radiata, basal ganglia, and thalami. MR angiogram; (e) shows mild atherosclerotic change with segmental narrowing of the anterior and middle cerebral arteries (white arrows); the internal carotid arteries and basilar artery are relatively normal.

\section{DISCUSSION}

Previous association studies on various risk factors such as hypertension, diabetes mellitus, cardiovascular disease, atrial fibrillation, carotid stenosis, and carotid intima-media thickness, and MRI-diagnosis of silent brain infarctions (SBI) were contradictory [4,5,9]. Regional cerebral blood flow in bilateral frontotemporal regions of brain measured by Xenon-133 inhalation method has been shown to reduce remarkably in neurologically normal adults with brain MRI evidence of silent lacunes [9]. But only less than half of the silent lacunes were seen on T1WI [9]. The autopsied data showed higher (81\%) frequency of SBI [3]. Recent advance in MRI technology improves the detection rate of SBI [4]. SBI was found more frequently than expected in subjects with no history of transient ischemic attack or stroke [4]. Hypertension was present in $72 \%$ of subjects with SBI $[4,6]$ that was in accord with our study. Prevalence of SBI even steeply increases after age of 65 years [4,6-8]. However, hypercholesterolemia, hyperglycemia (fasting 
plasma glucose $\geq 110 \mathrm{mg} / \mathrm{dL}$ ), and high hematocrit (Hct $\geq 50 \%$ ) or hypercoagulability were not risk factors of silent lacunes in several studies, but they were reported to be risk factors in another study [8,15]. Low-grade inflammation of cerebral vessel can be another risk factor of SBI or lacunes [16]. Among 40 patients with lacunes, $30 \%$ of cases showed single lesion on MRI [17] and these patients may have neuropsychological abnormality in certain aspect of cognitive domain, even though cognitive function in general remains normal [17]. Our data showed that simple neuropsychological tests such as MMSE, MMT, and Hachinski's score remained normal in patients with first-ever lacune, regardless whether they had or had not SBI [10-12]. A recent study working on more sophisticated neuropsychological tests performed at 1 month after acute lacunar infarction showed that $57.5 \%$ of patients had mild cognitive impairment (MCI) that was reflected on lower MMSE score (mean 28.4) [17]. MMSE score of 28 remains at the normal range of our healthy controls [11]. Furthermore, Grau and colleagues [17] included subjects with so called "atypical lacunes" in their study, and this part constituted more than a half of silent brain lacunes located in the striatothalamic region of the brain [17]. Atypical lacunes and pure motor hemiparesis accounted for most of the subjects with MCI [17]. Thalamic lesion constituted $44 \%$ of PS, $25 \%$ of PMH, and $25 \%$ of atypical lacune [17]. Nothing was known about unilaterality or bilaterality of the thalamic lesion and exact locations of the involvement of the different thalamic nuclei. A "typical thalamic lacune" usually located at the lateral tier of thalamic nucleus and frequently manifested with PS. However, a medially located thalamic infarction can be as small as a lacune, either unilateral or bilateral, and sometimes may be mistakenly considered as a "thalamic lacune" or an "atypical lacune". They are actually an ischemic stroke involving the paramedian mesencephalic arteries (PMAS) rather than the deep penetrating arteries of lacunar infarction [18]. PMAS is a branched or a trunk disease that frequently manifests itself with cognitive impairment, neurobehavioral dysfunction, and ocular signs [18]. It is a variant of the top-of-basilar artery syndrome. Therefore, more delicate test for various domains of cognition and higher brain functions might be needed to detect trivial abnormality in patients with lacunar stroke.

In addition, severe periventricular white matter (WM) change with higher grade $(\geq 2)$ of leuko-araiosis correlates with the presence of hypertension (13/18 patients, in contrast to $1 / 5$ in subjects with normal blood pressure) (Table 3). This is consistent with the previous studies [4-7]. Austrian Stroke Prevention Study (ASPS) looking at a community-based population on elderly normal subjects over 3-year follow-up [5] have shown that diastolic blood pressure and early-confluent white matter hyperintensities (WMH) at baseline MRI predicted best the white matter hyperintensity progression. This was also confirmed by a 6-year ASPS follow-up study [19]. Moreover, the WMH has been shown to associate with lower forced expiratory volume in 1 second (FEV1) and lower income of less than $\$ 50,000$ per year in CHS [6]. WMH progression did not associate with cognitive functioning [5], punctuate WM lesions are considered benign and not progressive, whereas early-confluent WM abnormalities are progressive within 6-year follow-up [19]. This is in contradiction to the CHS study that concluded that WM findings were associated with impaired cognitive and lower extremity functions [6].

Molecular markers of coagulation activation such as elevated prothrombin fragment, parameters for endothelial cell damage (thrombomodulin and von Willebrand factor), and lipoprotein A were significantly higher in patients with silent lacunes [15]. By contrast, a recently discovered plasma biomarker, SCUBE1, have been shown to associate with different forms of acute cerebral infarction and severity of ischemic stroke based on NIHSS score. The plasma level of SCUBE1 is remarkably

Table 3. Grading of leuko-araiosis (la) in 23 patients with "first-ever" lacunar infarction, high or normal blood pressure, and high or normal fasting plasma glucose.

\begin{tabular}{|c|c|c|c|c|c|c|c|c|c|}
\hline \multicolumn{5}{|c|}{ Grade } & \multicolumn{5}{|c|}{ Number $(\%)$ of Patients with } \\
\hline \multicolumn{5}{|c|}{ Lacunar Syndromes and Positive CT / MRI } & \multicolumn{5}{|c|}{ Associated Risk Factors } \\
\hline LA* & PMH (10) & SM (5) & $\mathrm{AH}(3)$ & $\mathrm{DCH}(3)$ & PS (2) & $\operatorname{HBP}(18)$ & NBP(5) & HPG(11) & NPG(12) \\
\hline $0 * *$ & $7 / 1$ & $2 / 1$ & $2 / 1$ & $1 / 0$ & $1 / 0$ & $0 * *$ & 3 & $0 * *$ & 3 \\
\hline 2 & $0 / 3$ & $0 / 1$ & $0 / 0$ & $0 / 0$ & $0 / 1$ & 4 & 1 & 2 & 3 \\
\hline 3 & $1 / 3$ & $1 / 1$ & $0 / 0$ & $1 / 2$ & $1 / 1$ & 7 & $0 * *$ & 6 & $1 * *$ \\
\hline 4 & $1 / 1$ & $1 / 1$ & $0 / 0$ & $0 / 0$ & $0 / 0$ & 2 & $0 * *$ & 2 & $0 * *$ \\
\hline
\end{tabular}

*LA: leuko-araiosis; PMH: pure motor hemiparesis; SM: sensorimotor syndrome; AH: ataxic hemiparesis; DCH: dysarthria-clumsy-hand syndrome; PS: pure sensory stroke; HBP: high blood pressure; NBP: normal blood pressure; HPG: high fasting plasma glucose; NPG: normal fasting plasma glucose.

grade 0 : absent of LA

grade 1: mild, LA confined to the frontal poles of lateral ventricles ("frontal caps")

grade 2: moderate, LA confined to the frontal and occipital poles of lateral ventricles ("dual caps")

grade 3: severe, LA involved the whole lateral ventricles ("dual caps" plus "rims")

grade 4: advanced, confluent LA lesions surround the centrum semiovale

$* * \mathrm{p}<0.05$ 
elevated in patients with acute infarction due to a trunkor branched-vessel disease, and slightly elevated or normal in patients with acute lacunar infarction [20]. Plasma level of oxidized small dense low-density lipoprotein (LDL-3) is an independent predictor of silent lacunar infarction [21].

In conclusion, evolving-stroke occurs in about onefifth of patients with "first-ever" acute lacunar infarction within the first 24 hours of stroke onset. Eighty-three percent of Chinese/Taiwanese patients with first-ever lacunar stroke had additional silent brain infarction on MRI. The presence of both hypertension and diabetes mellitus predicts the likelihood of high grade leukoaraiosis and additional SBI. Use of neuroimage studies in conjunction with scrutinized clinical assessments, monitoring stroke risk factors, and measurement of certain molecular or plasma biomarkers may be a future strategy for early diagnosis and treatment of patients at risk for silent brain lacunar infarctions.

\section{ACKNOWLEDGEMENTS}

The authors thank clinicians and technicians that contributed to the performance of brain CT and MRI examinations. Prof Rossane Harrigan is supported by a grant \#1R25RR019321 for Clinical Research Education and Career Development (CRECD) in Minority Institutions; and Prof Linda Chang is supported by a grant \#IU54 NS56883-03 for Imaging Studies in Neurotoxicity and Neurodevelopment, University of Hawaii Clinical Specialized Neuroscience Research Program (SNRP).

Conflict of interest: none to declare.

\section{REFERENCES}

[1] Fisher, C.M. (1982) Lacunar strokes and infarcts: A review. Neurology, 32(8), 871.

[2] Ropper, A.H. and Brown, R.H. (2005) Cerebrovascular diseases. In: Adams and Victor's Principles of Neurology, 8th Edition, McGraw-Hill Co., Inc., New York, 34, 682-684.

[3] Tuszynski, M.H., Petito, C.K. and Levy, D.E. (1989) Risk factors and clinical manifestations of pathologically verified lacunar infarctions. Stroke, 20(8), 990-999.

[4] Vermeer, S.E., Longstretch, W.I. and Kaudstaal, P.J. (2007) Silent brain infarcts: A systemic review. Lancet Neurology, 6(7), 611-619.

[5] Schmidt, R., Fazekas, F., Kapeller, P., Schmidt, H. and Hartung, H.P. (1999) MRI white matter hyperintensities: Three-year follow-up of the Austrian stroke prevention study. Neurology, 53(1), 132-139.

[6] Longstreth, W.T., Manolio, T.A., Arnold, A., Burke, G.L., Bryan, N., Jungreis, C.A., et al. (1996) Clinical correlates of white matter findings on cranial magnetic resonance imaging of 3301 elderly people: The cardiovascular health study. Stroke, 27(8), 1274-1282.

[7] Vermeer, S.E., Koudstaal, P.J., Oudkerk, M., Hofman, A. and Breteller, M.M.B. (2002) Prevalence and risk factors of silent brain infarcts in the population-based Rotterdam scan study. Stroke, 33(1), 21-25.
[8] Kwon, H.M., Kim, B.J., Lee, S.H., Choi, S.H., Oh, B.H. and Yoon, B.W. (2006) Metabolic syndrome as an independent risk factor of silent brain infarction in healthy people. Stroke, 37(2), 466-470.

[9] Kobayashi, S., Okada, K. and Yamashita, K. (1991) Incidence of silent lacunar lesion in normal adults and its relation to cerebral blood flow and risk factors. Stroke, 22(4), 1379-1383.

[10] Folstein, M.F., Folstein, S.E. and Mc Hugh, P.R. (1975) Mini mental state: A practical method for grading the cognitive state of patients for the clinician. Journal of Psychiatric Research, 12(3), 189-198.

[11] Thajeb, P., Thajeb, T. and Dai, D.F. (2007) Cross-cultural study using modified mini mental test (MMT) for healthy subjects and patients with various forms of vascular dementia. Journal of Clinical Neuroscience, 14(1), 236-241.

[12] Hachinski, V.C., Lassen, N.H. and Narshall, J. (1974) Multi-infarct dementia, a cause of mental deterioration in the elderly. Lancet, 2(7874), 207-210.

[13] Poirier, J. and Derouesne, C. (1985) The concept of cerebral lacunae from 1838 to the present (article in French, abstract in English). Review Neurology (Paris), 141(1), 3-17.

[14] Leifer, D., Buonanno. F.S. and Richardson, E.P.Jr. (1990) Clinicopathologic correlations of cranial magnetic resonance imaging of periventricular white matter. Neurology, 40(6), 911-918.

[15] Kario, K., Matsuo, T., Kobayashi, H., Asada, R. and Matsuo, M. (1996) Silent cerebral infaction is associated with hypercoagulability, endothelial cell damage, and high Lp(a) levels in elderly Japanese. Arteriosclerosis, Thrombosis, and Vascular Biology, 16(6), 734-741.

[16] Ishikawa, J., Tamura, Y., Hoshide, S., Eguchi, K., Ishikawa, S., Shimada, K. and Kario, K. (2007) Low-grade inflammation is a risk factor for clinical stroke events in addition to silent cerebral infarcts in Japanese older hypertensives: The Jichi medical school ABPM study, wave 1. Stroke, 38(3), 911-917.

[17] Grau-Olivares, M., Arboix, A., Bartre-Faz, D. and Junque, C. (2007) Neuropsychological abnormalities associated with lacunar infarction. Journal of Neurological Science, 257(1), 160-165.

[18] Thajeb, P., Thajeb, T. and Dai, D.F. (2007) Clinical analysis of first-ever acute ischemic stroke in the territory of paramedian mesencephalic arteries. Central European Journal of Medicine, 2(1), 37-46.

[19] Schmidt, R., Enzinger, C., Ropele, S., Schmidt, H. and Fazekas, F. (2003) Progression of cerebral white matter lesions: 6-year results of the Austrian stroke prevention study. Lancet, 361(9374), 2046-2048.

[20] Dai, D.F., Thajeb, P., Tu, C.F., Chiang, F.T., Chen, C.H., Yang, R.B. and Chen, J.J. (2008) Plasma concentration of SCUBE1, a novel platelet protein, is elevated in patients with acute coronary syndrome and ischemic stroke. Journal of the American College of Cardiology, 51(22), 21732180.

[21] Kato, T., Inoue, T., Yamagishi, S., Morooka, T., Okimoto, T. and Node, K. (2006) Low-density lipoprotein subfractions and the prevalence of silent lacunar infarction in subjects with essential hypertension. Hypertension Research, 29(5), 303-307. 